ARTÍCULO ORIGINAL

\title{
MALARIA PLACENTARIA SUBMICROSCÓPICA: HISTOPATOLOGÍA Y EXPRESIÓN DE MEDIADORES DE PROCESOS FISIOLÓGICOS
}

\author{
Carolina López-Guzmán (10) 1,a, Jaime Carmona-Fonseca (1) 2,b \\ 1 Grupo Salud y Comunidad-César Uribe Piedrahíta, Universidad de Antioquia, Medellín, Colombia. \\ 2 Facultad de Medicina, Universidad de Antioquia, Medellín, Colombia. \\ a Bacterióloga; maestra en Ciencias Básicas Biomédicas; ${ }^{\mathrm{b}}$ Médico cirujano; maestro en Microbiología
}

Este documento está basado, con modificaciones, en la tesis de maestría presentada por Carolina López-Guzmán para optar el respectivo título en la Corporación de Ciencias Básicas Biomédicas; Universidad de Antioquia, Medellín, Colombia; 2018. El tutor fue Jaime Carmona-Fonseca.

\section{RESUMEN}

Objetivos: Relacionar entre sí los eventos histopatológicos de malaria placentaria (MP), el comportamiento de células inmunitarias y la expresión de genes asociados a citoquinas, hipoxia, inflamación y angiogénesis en placentas con o sin infección plasmodial. Materiales y métodos: Diseño transversal, con tres grupos independientes. Las mujeres y sus placentas fueron captadas en 2009-2016, en los hospitales de Puerto Libertador y Tierralta, noroccidente de Colombia. El tamaño muestral se definió por conveniencia. El diagnóstico malárico se basó en PCR cuantitativa en tiempo real. Resultados: Se estudiaron 20 casos con MP por P. vivax (MP-V), 20 casos de MP por P. falciparum (MP-F) y $19 \sin$ MP; 95\% de los casos de MP son infección plasmodial placentaria submicroscópica (IPPS). Los tres grupos difieren en frecuencia y cantidad de eventos histopatológicos. Los mediadores de procesos fisiológicos presentaron diferencia significativa entre grupos, excepto IL-2, VEGF, VEGFR-1 y C5a. Conclusiones: Las placentas con infección difieren claramente de las no infectadas. $P$. vivax se comporta tan patógeno como $P$. falciparum. Se resalta la aproximación al abordaje integral del problema de MP. La infección plasmodial placentaria submicroscópica causa alteraciones tisulares y en mediadores fisiológicos como lo hace la infección microscópica, aunque probablemente en menor grado.

Palabras clave: Malaria; Plasmodium; Placenta; Histopatología; Patología; Mediador; Colombia (fuente: DeCS BIREME).

\section{SUBMICROSCOPIC PLACENTAL MALARIA: HISTOPATHOLOGY AND EXPRESSION OF PHYSIOLOGICAL PROCESS MEDIATORS}

\section{Citar como: López-Guzmán C, Carmona-Fonseca J. Malaria placentaria submicroscópica: histopatología y expresión de mediadores de procesos fisiológicos. Rev Peru Med Exp Salud Publica. 2020;37(2):220-8. doi: https://doi. org/10.17843/rpmesp.2020.372.4759}

Correspondencia: Jaime CarmonaFonseca, jaimecarmonaf@hotmail.com

Recibido: $21 / 08 / 2019$ Aprobado: 26/02/2020 En línea: 16/06/2020

\section{ABSTRACT}

Objetives: To relate histopathological events of placental malaria (PM), immune cell behavior and gene expression associated with cytokines, hypoxia, inflammation and angiogenesis in placentas with or without plasmodial infection. Materials and methods: Transversal design, with three independent groups. Women were recruited, and their placentas were collected in 2009-2016, in the hospitals of Puerto Libertador and Tierralta, northwestern Colombia. The sample size was defined by convenience. The malaria diagnosis was based on real-time quantitative PCR. Results: We studied 20 cases of PM by $P$. vivax (PM-V), 20 cases of PM by P. falciparum (PM-F) and 19 without PM; $95 \%$ of the cases of PM are submicroscopic placental plasmodial infection (SPPI). The three groups differ in frequency and number of histopathological events. Physiological process mediators showed significant difference between groups, except IL-2, VEGF, VEGFR-1 and C5a. Conclusions: Infected placentas are clearly different from uninfected ones. P. vivax behaves as pathogenic as P. falciparum. The approximation to the integral approach of the problem of PM is underlined. Submicroscopic placental plasmodial infection causes tissue and physiological mediator alterations as does microscopic infection, although probably to a lesser degree.

Keywords: Malaria; Plasmodium; Placenta; Histopathology; Pathology; Mediator; Colombia (source: MeSH NLM). 


\section{INTRODUCCIÓN}

La infección plasmodial placentaria submicroscópica (IPPS) por Plasmodium falciparum es frecuente en los países endémicos y contribuye al desarrollo de anemia materna y bajo peso al nacer ${ }^{(1)}$. La IPPS por $P$. vivax (IPPS-vivax) es casi desconocida en el mundo.

La técnica estándar para el diagnóstico de malaria en las zonas endémicas es la gota gruesa, que, por definición, no detecta las infecciones submicroscópicas, estas son usualmente asintomáticas. Estas dos condiciones, submicroscópicas y asintomáticas, ha mantenido a la IPPS en el olvido absoluto hasta comienzos del siglo xxi. Pero ya hay voces con fuertes argumentos que dicen, por ejemplo, que las infecciones asintomáticas tienen consecuencias importantes en la salud y la sociedad, y deben renombrarse como infecciones de malaria crónicas ${ }^{(2)}$.

La inmunopatogénesis de la malaria placentaria (MP) debe considerar que el embarazo es un estado fisiológico único en el que el sistema inmunológico materno debe proteger a la madre contra infecciones y otras noxas, $y$, a la vez, modular su respuesta inmune para evitar el rechazo del feto semialogénico ${ }^{(3)}$. La presencia de Plasmodium y sus productos (como hemozoína) en el tejido placentario, alteran en esencia el ambiente inmune que regula la placenta ${ }^{(4)}$.

En los casos de malaria gestacional y placentaria, el incremento de citoquinas TNF- $\alpha$, IFN- $\gamma$ e IL-10 se asocia con daño trofoblástico, bajo peso al nacer y prematurez ${ }^{(5)}$. La expresión de citoquinas proinflamatorias placentarias es alta en mujeres con MP y la expresión de citoquinas antiinflamatorias es baja ${ }^{(5-9)}$.

La MP altera la remodelación angiogénica ${ }^{(10,11)}$. Los infiltrados de células mononucleares y sus productos inflamatorios se asocian con bajo peso al nacer y es más breve y menos inflamatoria en mujeres multigrávidas que en primigrávidas. Estas diferencias pueden explicar los diferentes resultados que estas mujeres y su descendencia padecen ${ }^{(12-14)}$.

En el embarazo normal, son evidentes los procesos fisiológicos de apoptosis, inflamación, hipoxia, vasculogénesis y angiogénesis, entre otros, que están estrictamente controlados y en los que es decisivo el papel de señalizadores/mediadores de la comunicación celular (CC) ${ }^{(15-19)}$. El sistema de CC es un complejo entramado entre células, las cuales envían señales a otras, estas últimas con funciones receptoras, produciéndose una respuesta fisiológica y un cambio en la actividad celular. En la CC, los señalizadores de procesos fisiológicos o fisiopatológicos son muchos y tienen diverso origen (hormonas, factores de crecimiento, citoquinas, prostaglandinas, leucotrienos, etc.) ${ }^{(20)}$.

El objetivo del estudio fue determinar la asociación de la infección submicroscópica por $P$. vivax o $P$. falciparum con eventos histopatológicos, el comportamiento de células inmunitarias, expresión de genes asociados a citoquinas, hipoxia, angiogénesis e inflamación en placentas.

\section{MENSAJES CLAVE}

Motivación para realizar el estudio: Se tiene limitada evidencia sobre los efectos histopatológicos e inflamatorios de la infección placentaria submicroscópica por $P$. falciparum y $P$. vivax.

Principales hallazgos: Las placentas pertenecientes a mujeres residentes en la mayor zona endémica de Colombia, en el noroccidente del país, estaban afectadas por infección plasmodial submicroscópica (IPS), no detectada con gota gruesa, pero si con reacción en cadena de la polimerasa cuantitativa (qPCR). En la placenta, esta IPS causa daños tisulares y afecta mediadores de procesos como inflamación, hipoxia, angiogénesis, entre otros, comparada con placentas no infectadas. Tanto $P$. vivax como $P$. falciparum actúan como patógenos.

Implicancias: Hay que mejorar mucho las acciones de diagnóstico y tratamiento de la infección plasmodial gestacional en la consulta prenatal, que debe incluir obligatoriamente la práctica de gota gruesa en cada control.

\section{MATERIALES Y MÉTODOS}

\section{Diseño y población de estudio}

Se realizó un estudio transversal donde se compararon tres grupos independientes. El tamaño de la muestra se definió por conveniencia, según las muestras placentarias existentes en el banco de tejidos de nuestro grupo de investigación. Se seleccionaron 20 muestras con MP por vivax (MP-V) y 20 con MP por falciparum (MP-P) y como control se seleccionaron 19 muestras sin MP (MP-no). La prueba diagnóstica de infección plasmodial fue la reacción en cadena de la polimerasa cuantitativa en tiempo real (qPCR) en sangre placentaria.

Las mujeres y sus placentas fueron captadas en 2009 y 2016, en los hospitales de Puerto Libertador y Tierralta, municipios al sur del departamento de Córdoba, noroccidente de Colombia, en una región de alta endemicidad de malaria ${ }^{(21)}$. El sur de Córdoba, junto con Urabá Antioqueño y Bajo Cauca Antioqueño, conforman la región ecoepidemiológica que más casos de malaria genera anualmente en Colombia ${ }^{(21)}$.

Los criterios de inclusión fueron tener residencia permanente en esta región por lo menos durante el último año; no tener antecedentes de preeclampsia/eclampsia, enfermedad hipertensiva del embarazo, diabetes, VIH, y toxoplasmosis, rubeola, citomagalovirus, herpes simple (TORSCH); y tener parto con gestación de 36 a 41 semanas en alguno de los hospitales.

La edad gestacional se tomó de la historia clínica. Las mujeres del grupo control debían estar afebriles y las del grupo con infección plasmodial podían o no tener fiebre (solo $1 \%$ tuvo fiebre); asimismo deberían aceptar voluntariamente 
participar en el estudio. Los criterios de exclusión fueron retirar el consentimiento o la aparición de alguna complicación o enfermedad.

\section{Diagnóstico de malaria}

La sangre periférica materna se obtuvo en el momento del parto. La placenta se lavó con solución salina $(0,9 \%)$ y de su cara materna se tomaron muestras de sangre y tejido para diagnóstico (gota gruesa, qPCR, histopatología) como se ha indicado en otros informes ${ }^{(22-25)}$. Las muestras de gota gruesa fueron leídas por los investigadores y se definieron como negativas si 200 campos, con 100 aumentos, estuvieron libres de parásitos.

El ADN se extrajo con el método de saponina-Chelex y la qPCR ${ }^{(22,23)}$. El qPCR se ejecutó en la plataforma ABI 7500 FAST. Las muestras con un umbral de ciclo $(\mathrm{Ct})<45$ se analizaron en reacciones específicas de especies dúplex para P. falciparum y P. vivax ${ }^{(23)}$. Se usó la amplificación de los genes $18 \mathrm{~S}$ rRNA del ADN para la cuantificación. El número de copias de ADN se cuantificó a partir de la reacción específica del género frente a una curva estándar utilizando un plásmido que contiene un fragmento del gen $18 \mathrm{~S}$ de P. falciparum.

\section{Estudio histopatológico}

El tejido placentario se procesó según procedimientos estandarizados ${ }^{(24,25)}$. De cada placenta se tomaron dos fragmentos y de cada uno se hizo una placa para estudio histológico por microscopía de luz con procedimientos clásicos. Un fragmento procedió de un punto cercano a la inserción del cordón umbilical y el otro de la zona media (equidistante entre el cordón y el borde placentario). En total se leyeron 40 campos (20 por fragmento). La lectura fue cerrada frente a los resultados de gota gruesa y qPCR. Se usó aumento total de 400X para lectura histológica general. Se usó aumento total de $1000 \mathrm{X}$ para determinar la presencia de eritrocitos infectados (Ei) o hemozoína.

\section{Cuantificación de la expresión de genes asociados a señalizadores/mediadores}

Para cuantificar la expresión de genes asociados a señalizadores/mediadores y citoquinas en tejido placentario se usó un fragmento tisular preservado con RNA Later ${ }^{\circledast}$ (Qiagen) a $4{ }^{\circ} \mathrm{C}$. Los mediadores se agruparon así: proinflamatorios (IL-2, TNF, IFNy, COX-1, COX-2, C5a), antiinflamatorios (IL-10, IL-4), angiogénicos (VEGF, VEGFR-1) y de hipoxia (HIF).

Los diferentes señalizadores, excepto $\mathrm{C} 5 \mathrm{a}$, se midieron por cuantificación relativa del ARNm mediante qPCR. Se hizo cuantificación relativa para determinar los niveles de expresión de los mediadores del estudio en relación con los niveles de expresión del gen constitutivo y luego de obtener la relación entre el gen de interés y el gen constitutivo. Se aplicó el procedimiento de Pfaffl para determinar el delta-delta CT como aparece a continuación ${ }^{(26)}$ :
Expresión del gen $=\frac{\Delta \text { (Eficiencia del gen de interés })^{\mathrm{CT} \text { del gen de interés (CT control-CT muestra) }}}{\Delta(\text { Eficiencia del gen constitutivo })^{\mathrm{CT} \text { del gen constitutivo (CT control-CT muestra) }}}$

Para cuantificar la fracción C5a del complemento en las muestras de suero placentario, se usó el estuche comercial Human C5a Elisa Kit, siguiendo las recomendaciones del fabricante.

\section{Fuentes secundarias de información y grupos de estudio}

Después de la inclusión, se aplicó un cuestionario clínico-epidemiológico. La historia clínica fue usada como fuente de datos. Se evaluaron parturientas y sus placentas, repartidas en tres grupos: 19 en el grupo sin MP (MP-no) o grupo control, 20 en el grupo con MP-falciparum (MP-F) y 20 en el grupo con MP-vivax (MP-V).

\section{Análisis estadístico}

Se usaron los programas SPSS 18.0 y GraphPad Prism 5. Las decisiones sobre significación se tomaron con una probabilidad menor de 0,05. Se usó la prueba de Kolmogorov-Smirnov para evaluar la distribución normal de las variables cuantitativas. Para evaluar la homocedasticidad de las varianzas se usó la prueba de Levene. Se usó la prueba de Mann-Whitney para determinar si había diferencia entre dos grupos independientes. Se aplicó una prueba no paramétrica de Kruskal-Wallis para determinar si había diferencia entre tres grupos independientes, posteriormente, se usó la prueba de Dunn para identificar los pares de grupos que diferían.

\section{Consideraciones éticas}

El proyecto recibió aval del Comité de Bioética de la Sede de Investigación Universitaria SIU, Universidad de Antioquia (Medellín, Colombia) (acta de aprobación: 07-32-126; proyecto Colciencias código 111540820495, contrato: 238-2007).

\section{RESULTADOS}

El 95\% (38/40) de los casos de MP corresponden a infección submicroscópica. De las gestantes, 25\% (15/59) presentaron antecedentes de malaria durante la gestación actual.

El rango de la edad fue de 14 a 41 años y el promedio de los tres grupos fue similar (MP-no: 22; MP-F: 25; MP$\mathrm{V}: 23)$. La edad gestacional promedio fue de 38,6 semanas (rango 36 a 41). Las gestaciones previas tuvieron promedio de 2,8. El 36\% fueron primigestantes, el $20 \%$ secundigestantes y el $44 \%$ multigestantes (3 a 9). De los 59 partos, 5 fueron por cesárea.

Al momento del parto, la hemoglobina promedio fue de $11,1 \mathrm{~g} / \mathrm{dL}$. Las mujeres con MP tuvieron niveles menores de hemoglobina que las no infectadas $(10,86 \mathrm{~g} / \mathrm{dL}$ y $11,69 \mathrm{~g} / \mathrm{dL}$, respectivamente). La hemoglobina fue menor en el grupo 
de MP-V $(10,75 \mathrm{~g} / \mathrm{dL})$ que en MP-F $(10,97 \mathrm{~g} / \mathrm{dL})$, pero sin diferencia significativa $(\mathrm{p}=0,506)$. El promedio de peso neonatal en el grupo MP-no fue 2974 g; en MP-F fue 2852 $\mathrm{g}$ y en MP-V de $2737 \mathrm{~g}$. La diferencia de las medias de peso neonatal fue $237 \mathrm{~g}$ entre hijos del grupo MP-V y MP-no, y de $115 \mathrm{~g}$ entre hijos del grupo MP-V y MP-F.

El grupo control (MP-no) tuvo ausencia de necrosis y mayor frecuencia de aterosis, abrupcio y trombos que en los otros dos. El grupo MP-V presentó resultados similares a MP-F en aterosis, necrosis, infarto, depósitos de fibrina y trombos, pero menor cantidad de hemozoína y eritrocitos infectados. Los depósitos de fibrina estuvieron en todas las placentas. En el espacio intervelloso, $95-100 \%$ de las placentas presentaron hemorragia y 6 de cada 10 placentas mostraron trombos y calcificaciones. Existió diferencia significativa $(\mathrm{p}<0,05)$ entre los tres grupos en abrupcio, nodos sincitiales y hemozoína. El grupo de MP-F generó dicha diferencia. En la comparación por especie, no hubo diferencia significativa $(\mathrm{p}>0,05)$ (Tabla 1$)$.

En el grupo MP-no, la aterosis y el abrupcio presentan una cantidad escasa, con promedios de 1,3 y 2,2 eventos. En ese mismo orden; infarto y edema vellositarios tuvieron 9,1 y 8,7 eventos en promedio. En el mismo grupo MP-no, la cantidad de depósitos de fibrina y de nodos sincitiales es de 72,5 y 109, como promedio. Las placentas sin infección tienen 366 vellosidades, 1463 capilares y 3,97 capilares por vellosidad. El promedio de hemorragias fue 18 , mientras que los de trombos y calcificaciones fueron de dos (estuvieron en 60-70\% de ellas) (Tabla 2).

En los grupos con MP-F y MP-V la aparición de la infección placentaria reduce la cantidad de aterosis, de edema vellositario, de capilares por vellosidad, de hemorragias y trombos, pero aumenta la de infarto vellositario, calcificaciones y células inmunes. Solo unos pocos eventos presentan diferencia significativa (Tabla 2).

Las células inmunes estuvieron en todas las placentas, en los tres compartimentos, e independientemente de la infección. Las cantidades fueron diferentes, en el grupo MP-no se observó 9 células inmunes en decidua, 22 en vellosidad y 50 en espacio intervelloso; en el grupo MP-V se observó 58, 34 y 130, en el mismo orden; en el grupo MP-F se encontró la mayor cantidad de células inmunes: 68, 45 y 157, respectivamente (Tabla 2).

En la Tabla 3 se muestra la expresión de mediadores inflamatorios, angiogénicos e hipóxicos por grupo. La inflamación (COX-1, COX-2, IL-10, IFNy, TNF, C5a) fue significativamente mayor en MP. IL-2 e IL-4 variaron poco entre los tres grupos. C5a, aunque no mostró diferencia estadísticamente significativa entre los grupos, presentó valores mayores en MP-V comparado con otros dos grupos. En la angiogénesis, VEGF y VEGFR-1 no presentaron diferencia significativa entre grupos, pero su expresión aumentó en grupos en MP. En la hipoxia, HIF-1a tuvo diferencia significativa, con mayores valores en MP. En general, los mediadores que no presentaron diferencia significativa entre tres grupos fueron IL-2, VEGF, VEGFR-1 y C5a. Para VEGFR-1 y C5a, la alta variabilidad intragrupo es, probablemente, un factor que afecta la significación estadística al comparar los tres grupos.

En las Figuras 1, 2 y 3 se muestran las correlaciones lineales bivariadas entre eventos histológicos y mediadores de procesos. En el grupo sin infección, es claro el predominio de correlaciones significativas (CS) negativas y débiles

Tabla 1. Frecuencia de los hallazgos histológicos de la placenta según el grupo de estudio

\begin{tabular}{|c|c|c|c|c|c|c|c|c|}
\hline \multirow[t]{2}{*}{ Zona y evento } & \multicolumn{2}{|c|}{$\begin{array}{c}\text { Placenta sin malaria } \\
\text { (MP-no) }\end{array}$} & \multicolumn{2}{|c|}{$\begin{array}{l}\text { Malaria placentaria por } \\
\text { falciparum (MP-F) }\end{array}$} & \multicolumn{2}{|c|}{$\begin{array}{c}\text { Malaria placentaria por } \\
\text { vivax }(\mathrm{MP}-\mathrm{V})\end{array}$} & \multirow{2}{*}{$\begin{array}{c}\text { MP-No vs. MP-F } \\
\text { vs MP-V } \\
\text { Valor de p }\end{array}$} & \multirow{2}{*}{$\begin{array}{c}\text { MPV vs. MPF } \\
\text { Valor de p }\end{array}$} \\
\hline & $n=19$ & $\%$ & $\mathbf{n}=\mathbf{2 0}$ & $\%$ & $\mathbf{n}=\mathbf{2 0}$ & $\%$ & & \\
\hline \multicolumn{9}{|l|}{ Decidua } \\
\hline Aterosis & 10 & 53 & 6 & 30 & 7 & 35 & 0,317 & 0,736 \\
\hline Abrupcio & 12 & 63 & 8 & 40 & 3 & 15 & 0,009 & 0,077 \\
\hline Necrosis & 0 & 0 & 1 & 5 & 2 & 10 & 0,350 & 1,000 \\
\hline \multicolumn{9}{|l|}{ Vellosidad } \\
\hline Infarto & 16 & 84 & 18 & 90 & 19 & 95 & 0,537 & 0,696 \\
\hline Edema & 18 & 95 & 18 & 90 & 15 & 75 & 0,309 & 0,405 \\
\hline Nodos sincitiales & 19 & 100 & 20 & 100 & 15 & 75 & 0,005 & 0,056 \\
\hline Depósitos fibrinoides & 19 & 100 & 20 & 100 & 20 & 100 & 1,000 & 1,000 \\
\hline \multicolumn{9}{|l|}{ Espacio intervelloso } \\
\hline Trombos & 13 & 68 & 9 & 45 & 10 & 50 & 0,305 & 0,751 \\
\hline Calcificaciones & 12 & 63 & 13 & 65 & 12 & 60 & 0,947 & 0,744 \\
\hline Hemorragia & 19 & 100 & 19 & 95 & 19 & 95 & 1,000 & 1,000 \\
\hline Hemozoína & 0 & 0 & 10 & 50 & 6 & 30 & 0,002 & 0,197 \\
\hline Parásitos & 0 & 0 & 3 & 15 & 1 & 5 & 0,164 & 0,598 \\
\hline
\end{tabular}


Tabla 2. Magnitud de los eventos histológicos en placenta, según presencia de infección plasmodial

\begin{tabular}{|c|c|c|c|c|c|}
\hline \multirow[t]{2}{*}{ Zona y evento } & $\begin{array}{l}\text { Placenta sin malaria } \\
\text { (MP-no) }\end{array}$ & $\begin{array}{l}\text { Malaria placentaria por } \\
\text { falciparum (MP-F) }\end{array}$ & $\begin{array}{c}\text { Malaria } \\
\text { placentaria por } \\
\text { vivax }(\mathrm{MP}-\mathrm{V})\end{array}$ & $\begin{array}{l}\text { MP-No vs. MP-F } \\
\text { vs. MP-V }\end{array}$ & $\begin{array}{l}\text { MP-No vs. MP-V; } \\
\text { MP-F vs. MP-V }^{\text {a }}\end{array}$ \\
\hline & $\begin{array}{c}n=19 \\
\text { Media } \pm \text { DE }\end{array}$ & $\begin{array}{c}\mathbf{n}=\mathbf{2 0} \\
\text { Media } \pm \text { DE }\end{array}$ & $\begin{array}{c}n=20 \\
\text { Media } \pm \text { DE }\end{array}$ & Valor de $p$ & $\begin{array}{c}\text { Diferencias } \\
\text { significativas }\end{array}$ \\
\hline \multicolumn{6}{|l|}{ Decidua } \\
\hline Aterosis & $1,3 \pm 1,5$ & $0,5 \pm 1$ & $0,7 \pm 1,2$ & 0,198 & \\
\hline Abrupcio & $2,2 \pm 2,5$ & $2,7 \pm 4,0$ & $0,8 \pm 2,4$ & 0,031 & Sí; No \\
\hline Necrosis & $0,0 \pm 0,0$ & $0,1 \pm 0,2$ & $0,1 \pm 0,3$ & 0,371 & \\
\hline $\mathrm{CID}^{\mathrm{b}}$ & $9 \pm 6$ & $68 \pm 32$ & $58 \pm 30$ & 0,000 & Sí; No \\
\hline \multicolumn{6}{|l|}{ Vellosidad } \\
\hline Infarto & $9,1 \pm 8$ & $11,1 \pm 9$ & $15,8 \pm 11$ & 0,068 & \\
\hline Edema & $8,7 \pm 6$ & $6,9 \pm 5$ & $4,9 \pm 5$ & 0,095 & \\
\hline Depósitos fibrinoides & $72,5 \pm 22$ & $82,3 \pm 34$ & $65,5 \pm 20$ & 0,158 & \\
\hline Nodos sincitiales & $109 \pm 67$ & $150 \pm 60$ & $110 \pm 42$ & 0,037 & No; No \\
\hline Vellosidades & $366 \pm 44$ & $364 \pm 48$ & $326 \pm 61$ & 0,056 & \\
\hline Capilares & $1463 \pm 434$ & $1.446 \pm 634$ & $1,043 \pm 460$ & 0,018 & Sí; No \\
\hline Capilares/Vellosidad & $3,97 \pm 1,07$ & $3,87 \pm 1,35$ & $3,15 \pm 1,06$ & 0,050 & No; No \\
\hline $\mathrm{CIV}^{\mathrm{b}}$ & $22 \pm 11,3$ & $45 \pm 19,8$ & $34 \pm 15,8$ & 0,000 & No; No \\
\hline \multicolumn{6}{|l|}{ Espacio intervelloso } \\
\hline Hemorragias & $18 \pm 8$ & $13 \pm 8$ & $12 \pm 9$ & 0,090 & \\
\hline Trombo & $2,0 \pm 3$ & $1,1 \pm 2$ & $1,4 \pm 2$ & 0,345 & \\
\hline Calcificaciones & $1,9 \pm 1,8$ & $11,1 \pm 16,6$ & $16,6 \pm 22,6$ & 0,492 & \\
\hline CIEV $^{\mathrm{b}}$ & $50 \pm 24$ & $157 \pm 56$ & $130 \pm 54$ & 0,000 & Sí; No \\
\hline
\end{tabular}

a Indica si la prueba de Dunn mostró diferencia entre cada par de comparaciones (MP-no vs. MP-V; MP-V vs. MP-F) con p < 0,05.

b CID: células inmunes en decidua. CIV: células inmunes en vellosidad. CIEV: células inmunes en espacio intervelloso. DE: Desviación estándar

$(\mathrm{p}<0,10)$. Los depósitos de fibrinas, los nodos sincitiales y los trombos reúnen la mayor cantidad de CS. Las seis CS que tienen los depósitos fibrinoides se hacen con siete mediadores de procesos, tanto promotores de inflamación (COX-2 [pero no COX-1], C5a, IFNy [pero no TNF], VEGF) como de hipoxia (HIF) y angiogénesis (VEGF, pero no su receptor). Las cinco CS de los nodos sincitiales se hacen con tres de los mediadores que también las poseen con los depósi- tos fibrinoides (COX-2, HIF y VEGF) y con las citoquinas 2 (proinflamatoria) y 10 (antiinflamatoria). Las cuatro CS de los trombos suceden con los mediadores de inflamación TNF, IL-2, VEGF y el mediador de hipoxia HIF.

De otro lado, hay que resaltar las CS que establecen varios mediadores con algunos eventos histológicos. Es el caso de HIF, además de asociarse con los nodos sincitiales, los depósitos fribrinoides y los trombos, también lo está con in-

Tabla 3. Expresión de mediadores de inflamación, angiogénesis e hipoxia según grupo de estudio

\begin{tabular}{|c|c|c|c|c|c|}
\hline \multirow{2}{*}{ Mediador } & MP-no & MP-F & MP-V & $\begin{array}{c}\text { MP-No vs. MP-F vs. } \\
\text { MP-V }\end{array}$ & $\begin{array}{l}\text { MP-No vs. MP-V; } \\
\text { MP-V vs. MP-F }\end{array}$ \\
\hline & $\begin{array}{c}n=19 \\
\text { Media } \pm \text { DE }\end{array}$ & $\begin{array}{c}\mathbf{n}=\mathbf{2 0} \\
\text { Media } \pm \text { DE }\end{array}$ & $\begin{array}{c}\mathbf{n}=\mathbf{2 0} \\
\text { Media } \pm \text { DE }\end{array}$ & Valor de p & $\begin{array}{c}\text { Diferencias } \\
\text { significativas }\end{array}$ \\
\hline COX-1 & $0,9 \pm 0,3$ & $13,2 \pm 1,8$ & $13,6 \pm 1,1$ & 0,000 & Sí; No \\
\hline $\mathrm{COX}-2$ & $0,9 \pm 0,4$ & $5,9 \pm 0,5$ & $7,6 \pm 0,7$ & 0,000 & Sí; No \\
\hline IL-2 & $1,3 \pm 0,5$ & $1,0 \pm 0,2$ & $1,1 \pm 0,2$ & 0,413 & \\
\hline IL-4 & $1,3 \pm 0,4$ & $1,6 \pm 0,4$ & $1,6 \pm 0,3$ & 0,010 & No; No \\
\hline IL-10 & $1,2 \pm 0,3$ & $3,8 \pm 0,6$ & $3,8 \pm 0,4$ & 0,000 & Sí; No \\
\hline IFNy & $1,4 \pm 0,3$ & $10,6 \pm 1,6$ & $8,9 \pm 0,6$ & 0,000 & Sí; No \\
\hline TNF & $2,7 \pm 0,5$ & $10,2 \pm 0,7$ & $11,8 \pm 0,5$ & 0,000 & Sí; No \\
\hline HIF-1a & $0,6 \pm 0,5$ & $1,2 \pm 0,4$ & $1,1 \pm 0,3$ & 0,000 & Sí; No \\
\hline VEGF & $0,8 \pm 0,4$ & $1,0 \pm 0,1$ & $1,0 \pm 0,1$ & 0,179 & \\
\hline VEGFR-1 & $1,1 \pm 2,6$ & $2,4 \pm 4,9$ & $2,3 \pm 5,1$ & 0,891 & \\
\hline $\mathrm{C} 5 \mathrm{a}^{\mathrm{b}}$ & $77,7 \pm 13,9$ & $77,7 \pm 37,6$ & $94,7 \pm 26,8$ & 0,274 & \\
\hline
\end{tabular}

a Indica si la prueba de Dunn mostró diferencia entre cada par de comparaciones (MP-no vs MP-V; MPV vs. MP-F), con p < 0,05.

${ }^{\mathrm{b}}$ Para C5a se midió su concentración en sangre y no la expresión de su gen asociado. 


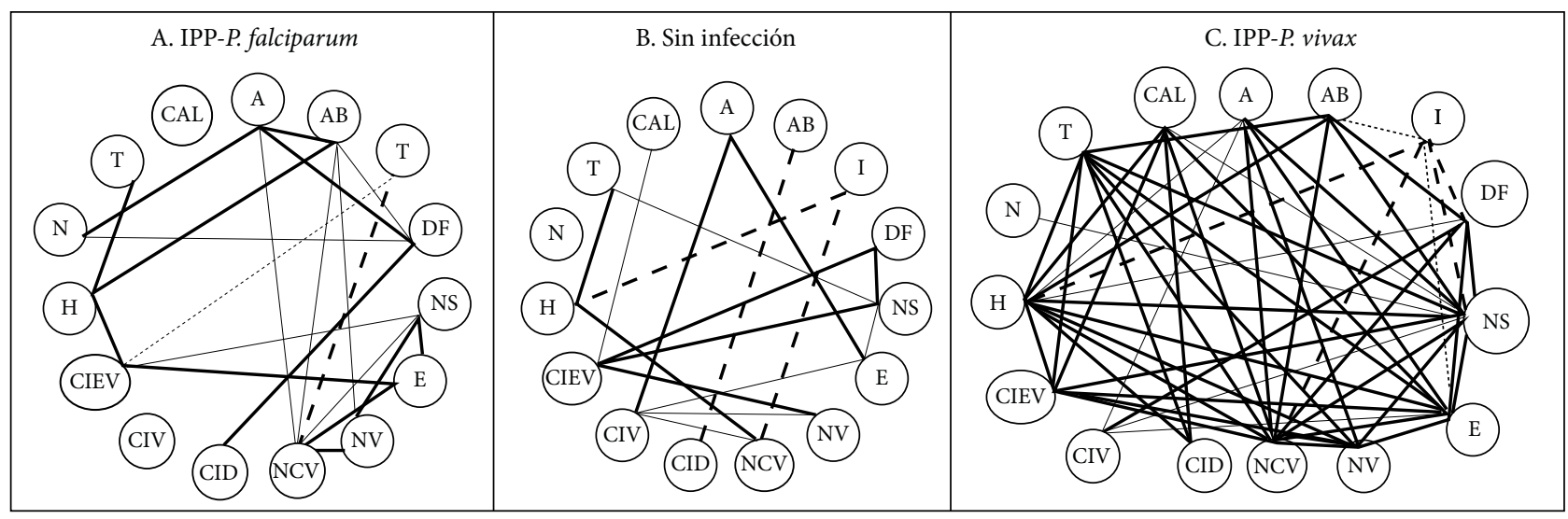

rho (+), p < 0,05; — rho (+), p $<0,10 ;--$ rho $(-), \mathrm{p}<0,05 ; \ldots-$ rho $(-), \mathrm{p}<0,10$

Cada evento ocupa siempre la misma posición en el plano.

Los círculos representan los hallazgos histológicos: A: aterosis, AB: abrupcio, I: infarto, DF: depósitos de fibrina, NS: nodos sincitiales, E: edema, NV: número de vellosidades, NCV: número de capilares por vellosidad, CID: células inmunes en decidua, CIV: células inmunes en vellosidad, CIEV: células inmunes en espacio intervelloso, H: hemorragia, N: necrosis, T: trombo, CAL: calcificaciones.

Las líneas sólidas/continuas y punteadas son correlaciones positivas y negativas, respectivamente.

El grosor o intensidad de la línea representa el grado de significación.

A. Grupo de placentas infectadas con P. falciparum. B. Grupo de placentas sin infección. C. Grupo de placentas infectadas por P. vivax.

En el grupo B (sin infección) todos los hallazgos histológicos tienen una correlación significativa (CS) excepto la necrosis (N) que fue el único hallazgo que no se encontró en estas placentas.

Es notoria la cantidad de CS en el grupo IPP-P. vivax respecto a los otros grupos. Además, la mayoría de las CS de MP-V son positivas y solo 4 del total de CS en este grupo son negativas y hacen referencia al infarto con $\mathrm{AB}, \mathrm{H}, \mathrm{NCV}, \mathrm{NS}$, DF y E.

Figura 1. Correlaciones lineales bivariadas significativas (CS) entre eventos histológicos placentarios

farto. TNF se relaciona con abrupcio, trombos y capilares vellositarios.

En el grupo MP-F, las CS se reducen y la polaridad cambia; ahora, los polos no son los nodos sincitiales, los depósitos fribrinoides y los trombos, sino el único polo que se destaca son los capilares vellositarios (tres CS: con TNF, HIF e IL-10). El TNF tuvo CS con edema, infarto, el número de vellosidades y el número de capilares por vellosidad; la IL-2 tuvo CS con abrupcio, aterosis, trombos y depósitos fibrinoides.

Para el grupo MP-V, las CS abundan como en la ausencia de infección, pero tiene otros centros: abrupcio, trombos,

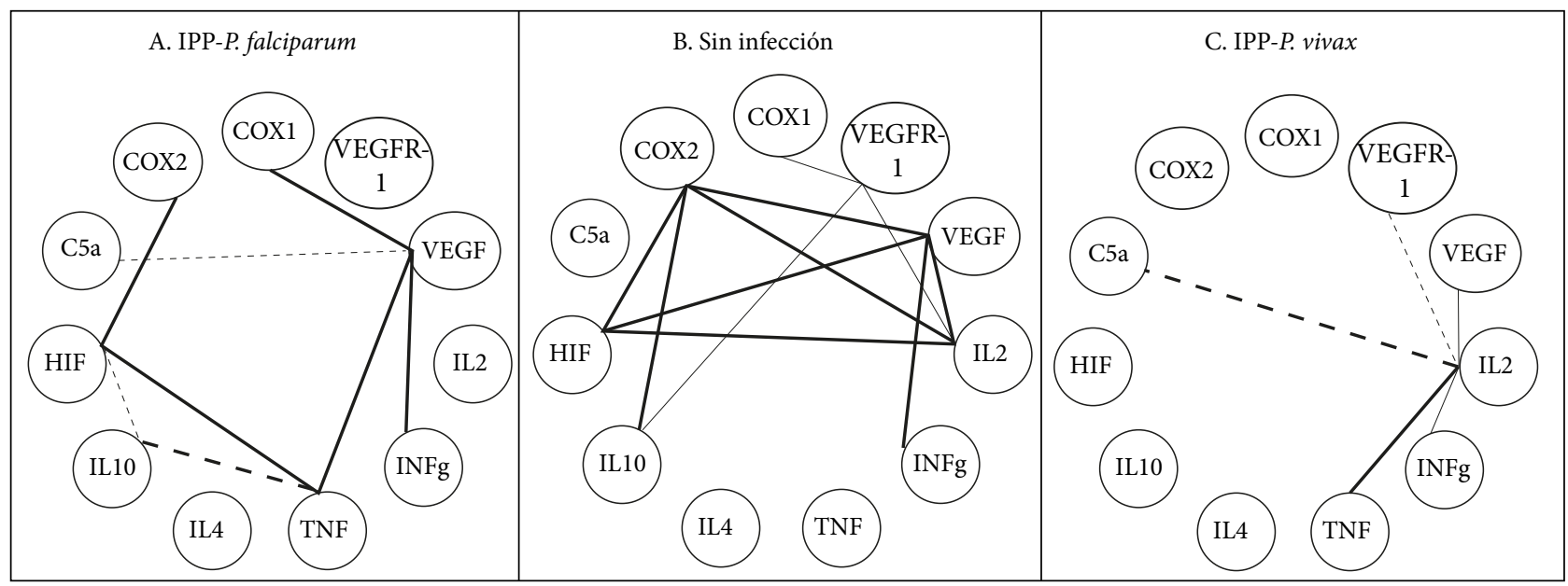

rho $(+), \mathrm{p}<0,05 ;-\operatorname{rho}(+), \mathrm{p}<0,10 ;--\operatorname{rho}(-), \mathrm{p}<0,05 ; \cdots-$ rho $(-), \mathrm{p}<0,10$

Los círculos representan los mediadores. Las líneas sólidas y punteadas son CS positivas y negativas, respectivamente. El grosor o intensidad de la línea representa el grado de significación.

En el grupo sin infección todas las CS que existen son positivas, se muestra que los mediadores de IL-2, COX-2, VEGF e HIF tienen la mayor cantidad de CS; seguido de VEGFR-1, IL-10 e IFNY. Es notoria la ausencia de CS de IL-4, TNF y C5a. En el grupo con infección es evidente que las CS cambian y aparecen CS negativas que no estaban en el grupo sin infección, en el grupo de IPP-P. vivax, aparecen menos CS y la IL-2 es el único centro de las CS existentes. En el grupo IPP-P. falciparum las CS son más descentralizadas. En ambos grupos con infección, el TNF y el C5a presentan CS, lo que no ocurrió en las placentas sin infección.

Figura 2. Correlaciones lineales bivariadas significativas (CS) entre mediadores de procesos placentarios de angiogénesis, inflamación e hipoxia 


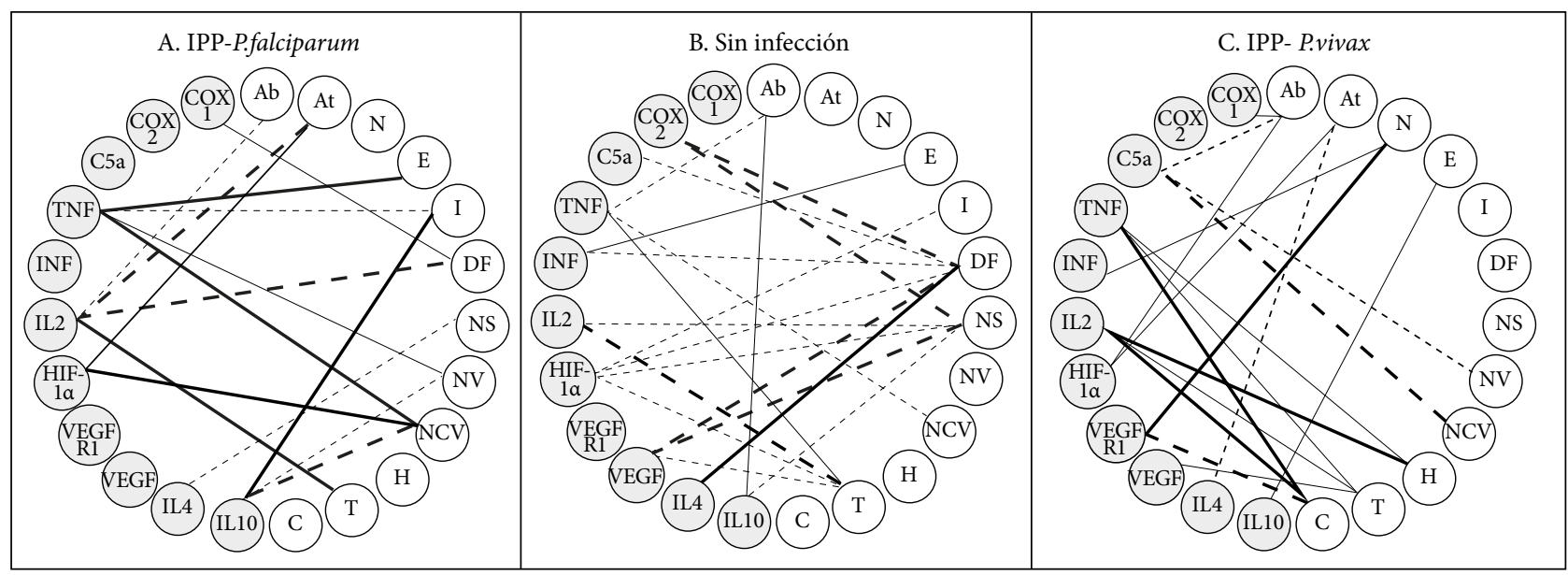

rho $(+), \mathrm{p}<0,05 ;-\operatorname{rho}(+), \mathrm{p}<0,10 ;-$ rho $(-), \mathrm{p}<0,05 ;-\cdots$ rho $(-), \mathrm{p}<0,10$

Los círculos representan los mediadores. Las líneas sólidas y punteadas son correlaciones significativas (CS) positivas y negativas, respectivamente. El grosor o intensidad de la línea representa el grado de significación.

En el grupo sin infección todas las CS que existen son positivas, se muestra que los mediadores de IL-2, COX-2, VEGF e HIF tienen la mayor cantidad de CS; seguido de VEGFR-1, IL-10 e IFNy. Es notoria la ausencia de CS de IL-4, TNF y C5a. En los grupos con infección es evidente que las CS cambian y aparecen CS negativas que no estaban en el grupo sin infección. En IPP-P. vivax, aparecen menos CS y la IL-2 es el único centro de las CS existentes. En el grupo IPP-P. falciparum las CS son más descentralizadas. En ambos grupos con infección, el TNF y el C5a presentan CS, lo que no ocurrió en las placentas sin infección.

Figura 3. Correlaciones lineales entre eventos histológicos y mediadores de procesos en placenta

calcificaciones. Las CS del abrupcio se dan con COX-1, C5a e HIF-1 1 ; las de trombos y calcificaciones con el TNF e IL2. Además los trombos se asocian a VEGF, en cambio, las calcificaciones se relacionan con VEGFR-1.

\section{DISCUSIÓN}

En el presente trabajo se hallaron diferencias importantes en los efectos placentarios de las dos especies plasmodiales evaluadas. La cantidad de abrupcio, nodos sincitiales, vellosidades, capilares y capilares por vellosidad es significativamente menor cuando hay $P$. vivax, respecto a P. falciparum. Por otra parte, P. vivax comparado con las placentas sin infección, generó más infarto y menos abrupcio, edema, vellosidades, capilares y capilares por vellosidad. La MP-IPPS debida a $P$. falciparum tiene pruebas sobre su naturaleza patógena en gestantes y sus hijos ${ }^{(1)}$. Inclusive en estudios en la misma zona geográfica en donde se hizo este trabajo ${ }^{(27-29)}$, la infección submicroscópica placentaria por $P$. vivax ocasiona efectos nocivos.

Las comparaciones del grupo de $P$. vivax contra $P$. falciparum o contra el grupo sin infección apoyan fuertemente la capacidad patogénica placentaria de $P$. vivax. Estos hallazgos sientan bases para explicar sus efectos por un mecanismo que no necesariamente involucra la citoadhesión de eritrocitos infectados al tejido placentario, como se da en infecciones por $P$. falciparum.

Existe escasa información sobre las correlaciones entre eventos histológicos y mediadores de procesos. Cuando no hay infección, las CS entre eventos histológicos y mediado- res de procesos son predominantemente menores al $10 \%$. Esto se puede interpretar como un estado homeostático, en el que no predomina una respuesta específica, sino que todas ellas se encuentran activas, pero en estados basales y equilibrados. En caso contrario la gestación estaría en riesgo.

Cuando existe infección plasmodial, el panorama de las CS cambia en forma radical, tanto cuando se trata de infección plasmodial placentaria por $P$. falciparum (IPP-F), pero sobre todo en infección plasmodial placentaria por P. vivax (IPP-V). Las CS que había en el estado de no infección desaparecen y surgen otras nuevas en las infecciones plasmodiales. Los centros o polos de CS que eran los nodos sincitiales y los depósitos fibrinoides desaparecen y son reemplazados por polos que ahora pertenecen a los mediadores de procesos, especialmente de inflamación. La IL-2, el TNF e la IL-10 son polos en IPP-F, mientras que la IL-2, el TNF y el C5a lo son en IPP-V. En ausencia de infección, las vellosidades y los capilares están prácticamente sin CS, cuando aparece infección ellos surgen como polos. Todo esto expresaría el estado inflamatorio exacerbado de la placenta como consecuencia de la infección.

En el presente estudio no se midieron hormonas en ninguno de los trimestres de la gestación. La progesterona, estrógenos, andrógenos y glucocorticoides están involucrados en la gestación desde la implantación hasta el parto; su biosíntesis y su metabolismo son el resultado de vías complejas que involucran al feto, la placenta y la madre ${ }^{\left({ }^{30}\right)}$. Muchas hormonas sexuales tienen clara interacción con el sistema inmune y muchos mediadores de procesos fisiológicos también interactúan con tal sistema. Existe, entonces, una compleja red fisiológica entre hormonas, mediadores de procesos fisiológicos y sistema inmune. 
Entre las fortalezas del estudio está su aproximación integral a la MP, abordando la relación entre eventos histopatológicos y mediadores de inflamación, angiogénesis e hipoxia. A nuestro conocimientos estas relaciones no han sido estudiadas. Se tuvo un grupo control bien definido, el cual fue negativo para Plasmodium según técnica altamente sensible y específica (qPCR), sin presencia de TORCHS, $\mathrm{VIH}$, eclampsia-preeclampsia, y diabetes. Sin embargo, no se pudo descartar la presencia de parásitos intestinales y desnutrición.

Entre las limitaciones señalamos el pequeño tamaño muestral y la selección por conveniencia, generado por las condiciones de tiempo y dinero disponibles. Los mediadores evaluados representan básicamente el proceso inflamatorio de la placenta a término, otros mediadores de procesos no fueron medidos suficientemente. El análisis de las correlaciones entre hallazgos histológicos y mediadores de procesos es superficial porque la información disponible es escasa o inexistente.

En conclusión, cuando ocurre IPPS hay cambios tisulares placentarios y cambios en la expresión de mediadores del pro-

\section{REFERENCIAS BIBLIOGRÁFICAS}

1. Arango E, Maestre A, Carmona-Fonseca J. Efecto de la infección submicroscópica o policlonal de Plasmdium falciparum sobre la madre y el producto de la gestación. Revisión sistemática. Rev Bras Epidemiol. 2010;13(3):373-86. doi: 10.1590/s1415-790x2010000300002.

2. Chen I, Clarke SE, Gosling R, Hamainza B, Killeen G, Magill A, O’Meara W, Price RN, Riley EM. "Asymptomatic" malaria: a chronic and debilitating infection that should be treated. PLoS Med 13(1): e1001942.

3. Mor G, Cardenas I. The immune system in pregnancy: a unique complexity. Am J Reprod Immunol. 2010;63(6):425-33.

4. Rogerson SJ, Hviid L, Duffy PE, Leke RF, Taylor DW. Malaria in pregnancy: pathogenesis and immunity. Lancet Infect Dis. 2007;7(2):105-17.

5. Menendez C, Ordi J, Ismail MR, Ventura PJ, Aponte JJ, Kahigwa E, et al. The impact of placental malaria on gestational age and birth weight. J Infect Dis. 2000;181(5):1740-5.

6. Chaisavaneeyakorn S, Lucchi N, Abramowsky C, Othoro C, Chaiyaroj SC, Shi YP, et al. Immunohistological characterization of macrophage migration inhibitory factor expression in Plasmodium falciparum-infected placentas. Infect Immun. 2005;73(6):3287-93.

7. Suguitan AL, Leke RG, Fouda G, Zhou A, Thuita L, Metenou S, et al. Changes in the levels of chemokines and cytokines in the placentas of women with Plasmodium falciparum malaria. J Infect Dis. 2003;188(7):1074-82.

8. Lucchi NW, Koopman R, Peterson DS, Moore JM. Plasmodium falciparum-infected red blood cells selected for binding to cultured syncytiotrophoblast bind to chondroitin sulfate $A$ and induce tyrosine phosphorylation in the syncytiotrophoblast. Placenta. 2006;27(4-5):384-94.

9. Salih MM, Mohammed AH, Mohmmed AA, Adam GK, Elbashir MI, Adam I. Monocytes and macrophages and placental malaria infections in an area of unstable malaria transmission in eastern Sudan. Diagn Pathol. 2011;6:83.

10. Hromatka BS1, Ngeleza S, Adibi JJ, Niles RK, Tshefu AK, Fisher SJ. Histopathologies, immunolocalization, and a glycan binding screen provide insights into Plasmodium falciparum interactions with the human placenta. Biol Reprod. 2013;88(6):154. doi: 10.1095/biolreprod.112.106195.

11. Souza RM, Ataíde R, Dombrowski JG, Ippólito V, Aitken EH, Valle SN et al. Placental histopathological changes associated with Plas- ceso inflamatorio, tanto si el agente causal es $P$. falciparum como si es $P$. vivax. Si es que el parásito es patógeno para la placenta, cabe esperar que cuando las parasitemias se incrementen, más fuertes serán sus efectos. La infección placentaria por $P$. vivax contribuye al aumento de hallazgos histológicos placentarios asociados con deterioro y daño del tejido. La alteración de la estructura placentaria estuvo principalmente asociada a la disminución de vellosidades y del número de capilares por vellosidad, así como con el aumento de lesiones degenerativas isquémicas asociados con calcificaciones e infarto. Estos resultados podrían correlacionarse con disminución del peso al nacer y con disminución en los niveles de hemoglobina.

Contribuciones de los autores: CLG y JCF concibieron y ejecutaron todas las fases de la investigación; asimismo, analizaron e interpretaron los datos y elaboraron el artículo.

Fuentes de financiamiento: Colciencias proyecto 111577757051 , contrato 755-2017; Estrategia de Sostenibilidad Codi-Universidad de Antioquia 2016-2017 (código ES-84160127); Universidad de Antioquia.

Conflictos de interés: Los autores declaran no tener conflictos de interés en la publicación del artículo. modium vivax infection during pregnancy. PLoS Negl Trop Dis. 2013;7(2):e2071. doi: 10.1371/journal.pntd.0002071.

12. Duffy PE. Plasmodium in the placenta: parasites, parity, protection, prevention and possibly preeclampsia. Parasitology. 2007;134(Pt 13):1877-81.

13. Hartman TK, Rogerson SJ, Fischer PR. The impact of maternal malaria on newborns. Ann Trop Paediatr. 2010;30(4):271-82.

14. Kabyemela ER, Fried M, Kurtis JD, Mutabingwa TK, Duffy PE. Fetal responses during placental malaria modify the risk of low birth weight. Infect Immun. 2008;76(4):1527-34.

15. Lucchi NW, Peterson DS, Moore JM. Immunologic activation of human syncytiotrophoblast by Plasmodium falciparum. Malar J. 2008;7:42. doi: 10.1186/1475-2875-7-42.

16. Valdivia-Silva JE, González-Altamirano C, López-Molina K, Lazo-Velásquez JC, García-Zepeda E. Relación de la expresión del factor inducido por hipoxia-2 $\alpha$ (HIF-2 $\alpha$ ) y sVEGF-R1/sFlt-1: implicación en la fisiopatología de preeclampsia. Clin Invest Ginecol Obstet. 2008;35(1):11-23.

17. Soeters PB, Grimble RF.The conditional role of inflammation in pregnancy and cancer. Clin Nutr. 2013;32(3):460-5.

18. Laakkonen JP, Lähteenvuo J, Jauhiainen S, Heikura T, Ylä-Herttuala S. Beyond endothelial cells: Vascular endothelial growth factors in heart, vascular anomalies and placenta. Vascul Pharmacol. 2019;112:91-101. doi: 10.1016/j.vph.2018.10.005.

19. Melincovici CS, Boşca AB, Şuşman $S$, Mărginean M, Mihu C, Istrate $\mathrm{M}$ et al. Vascular endothelial growth factor (VEGF) - key factor in normal and pathological angiogenesis. Rom J Morphol Embryol. 2018;59(2):455-467.

20. Biocancer. Tipos y moléculas de señalización. Acceso: 7 junio 2019. Disponible en: http://www.biocancer.com/journal/1101/11-tipos-y-moleculas-de-senalizacion.

21. Carmona-Fonseca J. Región "Urabá Antioqueño-Cuencas altas de ríos Sinú y San Jorge-Bajo Cauca Antioqueño": "guarida” del paludismo colombiano. Rev Salud UIS. 2017;49(4):577-589.

22. Plowe CV, Djimde A, Bouare M, Doumbo O, Wellems TE. Pyrimethamine and proguanil resistance-conferring mutations in Plasmodium 
falciparum dihydrofolate reductase: polymerase chain reaction methods for surveillance in Africa. Am J Trop Med Hyg. 1995; 52(6):565-8. doi: 10.4269/ajtmh.1995.52.565.

23. Shokoples SE, Ndao M, Kowalewska-Grochowska K, Yanow SK. Multiplexed real-time PCR assay for discrimination of Plasmodium species with improved sensitivity for mixed infections. J Clin Microbiol. 2009; 47(4):975-80. doi: 10.1128/JCM.01858-08.

24. Carmona-Fonseca J, Arango E, Maestre A. Placental malaria in Colombia: histopathologic findings in Plasmodium vivax and P.falciparum infections. Am J Trop Med Hyg. 2013; 88(6):1093-101. doi: 10.4269/ajtmh.12-0363.

25. Agudelo-García OM, Arango-Flórez EM, Carmona-Fonseca J. Submicroscopic and asymptomatic congenital infection by Plasmodium vivax or $P$. falciparum in Colombia: 37 cases with placental histopathology and cytokine profile in maternal and placental blood. J Trop Med. 2017; 2017:368078.

26. Pfaffl MW. A new mathematical model for relative quantification in real-time RT-PCR. Nucleic Acids Res. 2001;29(9):e45. doi: 10.1093/ nar/29.9.e45.
27. Carmona-Fonseca J, Agudelo O, Arango E. Asymptomatic plasmodial infection in Colombian pregnant women. Acta Trop. 2017; 172:97-101. doi: 10.1016/j.actatropica.2017.04.030.

28. Agudelo O, Aristizabal B, Yanow S, Arango E, Carmona-Fonseca J, Maestre A. Submicroscopic infection of placenta by Plasmodium produces Th1/Th2 cytokine imbalance, inflammation and hypoxia in women from Northwest Colombia. Malar J. 2014;13:122.

29. Agudelo-García OM. Malaria placentaria submicroscópica por Plasmodium vivax o Plasmodium falciparum: histopatología, células inmunitarias y expresión de genes asociados a hipoxia, apoptosis e inflamación. Trabajo de investigación para optar el título de Doctor en Ciencias Básicas Biomédicas. Corporación Académica Ciencias Básicas Biomédicas, Grupo Salud y Comunidad-César Uribe Piedrahíta, Universidad de Antioquia; Medellín, octubre 2017.

30. Morel Y, Roucher F, Plotton I, Goursaud C, Tardy V, Mallet D. Evolution of steroids during pregnancy: Maternal, placental and fetal synthesis. Ann Endocrinol (Paris). 2016;77(2):82-9. doi: 10.1016/j.ando.2016.04.023. 